\title{
EXTREMALS OF THE GENERALIZED EULER-BERNOULLI ENERGY AND APPLICATIONS
}

\author{
ÓSCAR J. GARAY
}

Communicated by Ivaïlo M. Mladenov

\begin{abstract}
In this survey we describe a general method to deal with the variational problem associated to the generalized elastic curves, paying special attention to closed critical curves in real space forms due to its special geometric significance. We illustrate the method by studying particular choices of this energy in some more detail. Finally, we will review also some interesting applications of generalized elasticae to other higher dimensional variational problems in Physics, Biophysics and the Theory of Submanifolds.
\end{abstract}

\section{Introduction}

In $1691 \mathrm{~J}$. Bernoulli posed the problem of the elastic beam and three years later he published his own solution. However, Huygens criticized his work for not showing all the possible solutions. In 1742, D. Bernoulli proposed to minimize the squared radius of curvature in order to determine the shape of an elastic rod subject to pressure at both ends. Thus, following the D. Bernoulli's simple geometric model, an elastic curve (also, elastica) is a minimizer of the bending energy

$$
\mathcal{F}_{\lambda}(\gamma)=\int_{\gamma}\left(\kappa^{2}+\lambda\right) \mathrm{d} s
$$

where $\kappa$ represent the curvature of the curve $\gamma$ and $\lambda$ corresponds to a constraint on its length. When $\lambda=0$, critical points of bending energy are called free elastic curves or, simply, elasticae. In 1743, L. Euler determined the plane elastic curves [23] (explicit expressions for them can be found in [22]). Elastic curves in $\mathbb{R}^{3}$ were first considered by J. Radon 1910 and R. Irrgang 1933. More recently, J. Langer and D. Singer [38], [40], [41], [59], have made significant contributions to the subject. The Hamiltonian theory has been developed by V. Jurdjevic in [30] and a different approach has also been proposed by R. Bryant and Ph. Griffiths in [18]. This work deals with the study of curves which are essentially one-dimensional 
objects with negligible thickness. Consideration of the effect of thickness on the elastic energy lead to the Kirchhoff elastic rod model (see, for example, [32], [41] and [59]).

Closed elastic curves have a special geometric relevance, what have attracted the attention of many authors over the years. J. Langer and D. Singer in 1987 and N. Koiso in 1993, showed by different methods that there exist closed elastic curves of a given length in a compact Riemannian manifold. Moreover, J. Langer and D. Singer classified the closed free elastic curves in two-dimensional space forms [38]. They showed also that there exist a countable family of closed elastic curves in $\mathbb{R}^{3}$ [39] (for a recent survey, see [59]). Closed elasticae in $\mathbb{S}^{3}(1)$ were studied by J. Arroyo, O. Garay and J. Mencía in [11].

In this paper, we are interested in a generalization of the bending energy functional which was first considered, restricted to curves in $\mathbb{R}^{3}$, by G. Bliss 1907, J. Radon 1910 and by W. Blaschke 1930. We want to study the variational problem associated to curvature energy functionals of the generalized Euler-Bernoulli energy family

$$
\mathcal{F}(\gamma)=\int_{\gamma} P(\kappa) \mathrm{d} s
$$

acting on certain spaces of curves of a Riemannian manifold (as usual, here $\kappa$ denotes the curvature function of $\gamma$ and $P(\kappa)$ is a smooth function).

For suitable choices of $P$ critical points of (2) include geodesics, classical elasticae, elasticae circular at rest, closed elasticae enclosing a fixed area,...etc. We shall see that these family of functionals have also interesting applications in Physics to the construction of models of relativistic particles (massive or massless) and $p$-branes; in Biophysics to the study of membranes and vesicles; and, in Mathematics to the Theory of Submanifolds, where they can be used, for instance, to build algorithms which provide efficient ways to obtain new examples of ChenWillmore submanifolds.

Since, depending on whether $\frac{\mathrm{d} P^{\prime}}{\mathrm{d} s}=0$ or not, the techniques that are normally used are different, we are going to consider these two cases separately: (1) Order one functionals, where the smooth function $P$ is assumed to satisfy $\frac{\mathrm{d} P^{\prime}}{\mathrm{d} s}=0$; and (2) Higher order functionals, where $P$ verifies $\frac{\mathrm{d} P^{\prime}}{\mathrm{d} s} \neq 0$. But first, some notation and useful tools are reminded in the next section. 


\section{Preliminaries}

Let us assume that $\mathbb{M}^{n} n$-dimensional Riemannian manifold with metric $\langle$, which will be denoted by $\mathbb{M}^{n}(G)$ when $n$-dimensional is a real space form with constant curvature $G$. As usual, the Levi-Civita connection and curvature tensor of $\mathbb{M}^{n}$ are denoted by $\nabla$ and $R$, respectively.

In the following, $\mathcal{H}$ will represent a certain space of curves, $\gamma: \mathbb{I}=[0,1] \rightarrow \mathbb{M}^{n}$, satisfying suitable boundary conditions. Curves $\mathcal{H}$ will satisfy at least: (1) $\gamma \in$ $C^{4}(\mathbb{I})$; (2) $\gamma$ is immersed in $\mathbb{M}^{n}$, that is, $\frac{\partial \gamma}{\partial t} \neq 0$; and (3) there is a well defined normal vector on $\gamma$ (for instance, if $n=2$ and $\mathbb{M}^{2}$ is orientable, or if $\frac{\partial^{2} \gamma}{\partial t^{2}} \neq 0$ ). We shall denote by $\Omega$ the space of smooth closed curves.

Now, given an immersed curve of $\mathbb{M}^{n}, \gamma: \mathbb{I}=[0,1] \rightarrow \mathbb{M}^{n}$, its tangent vector will be $V(t)=\frac{\partial \gamma}{\partial t}=\gamma^{\prime}(t)$ and, therefore, $v(t)=\langle V, V\rangle^{\frac{1}{2}}$ will denote the speed of $\gamma$. The standard symbols $T(t), N(t)$ and $B(t)$ will be used to represent the Frenet Frame associated to $\gamma$. Then, the curvature and torsion of $\gamma$ are defined respectively by $\kappa(t)=\left\|\nabla_{T} T\right\|$ and $\tau(t)=\left\|\nabla_{T} B\right\|(\kappa$ will be the oriented curvature if $\gamma$ lies in an oriented surface $\mathbb{M}^{2}$ ).

On the other hand, if $\gamma_{w}(t)=\gamma(w, t):(-\varepsilon, \varepsilon) \times \mathbb{I} \rightarrow \mathbb{M}^{n}$ is a variation of $\gamma(t)=\gamma(0, t)$, then $W=W(t)=\frac{\partial \gamma}{\partial w}(0, t)$ will denote the variational vector field along the curve. The letter $s \in[0, L]$ will be employed for the arclength parameter of $\gamma$ ( $L$ is the length of the curve).

Finally, a vector field $W$ defined on regular curve $\gamma$ immersed in $\mathbb{M}^{3}(G)$, is called a Killing field along $\gamma$, if for any variation of $\gamma$ with variational field $W$, we have

$$
\frac{\partial v}{\partial w}=\frac{\partial \kappa}{\partial w}=\frac{\partial \tau}{\partial w}=0 .
$$

J. Langer and D. Singer [38] have shown that a Killing field along $\gamma$ of a real space form $\mathbb{M}^{3}(G)$ is the restriction of a Killing field defined on the whole manifold.

\subsection{A Useful Tool I: Hopf Cylinders}

We consider the well known Hopf map $\pi: \mathbb{S}^{3}(1) \rightarrow \mathbb{S}^{2}\left(\frac{1}{2}\right)$ acting on the threesphere [28]. It is a Riemannian submersion when the base space $\mathbb{S}^{2}\left(\frac{1}{2}\right)$ is chosen to have radius $\frac{1}{2}$. If $\beta$ is a curve in $\mathbb{S}^{2}\left(\frac{1}{2}\right)$, then $\bar{\beta}$ will denote a horizontal lift of $\beta$ to $\mathbb{S}^{3}(1)$ via $\pi$. For any curve $\beta(s)$ in $\mathbb{S}^{2}\left(\frac{1}{2}\right)$, its complete lift to $\mathbb{S}^{3}(1)$ via $\pi$

$$
\mathbf{T}_{\beta}=\pi^{-1}(\beta)=\left\{\mathrm{e}^{\mathrm{i} t} \cdot \bar{\beta}(s) ;(s, t) \in \mathbb{R}^{2}\right\}
$$


will be called the Hopf Cylinder shaped on $\beta$. By using the fact that the map $\phi=\phi(z, t): \mathbb{R}^{2} \rightarrow \mathbf{T}_{\beta}$ defined by

$$
\phi(z, t)=\mathrm{e}^{\mathrm{i} z} \cdot \bar{\beta}(t)=\cos z \bar{\beta}(t)+\sin z \eta(t)
$$

is as a covering map, it is not difficult to check the following well known facts:

- Hopf cylinders $\mathbf{T}_{\beta}$ are flat surfaces with the induced metric from $\mathbb{S}^{3}(1)$. A Hopf cylinder $\mathbf{T}_{\beta}$ is embedded in $\mathbb{S}^{3}(1)$ if $\beta$ is a simple curve in $\mathbb{S}^{2}\left(\frac{1}{2}\right)$.

- If $\beta$ is a closed curve, then the Hopf tube $\mathbf{T}_{\beta}$ is a flat torus whose isometry type depends on the length and enclosed area of $\beta$, i.e., $\mathbf{T}_{\beta}$ is isometric to $\mathbb{R}^{2} / \Gamma$, where $\Gamma$ is the lattice in $\mathbb{R}^{2}$ spanned by $(2 A, L)$ and $(2 \pi, 0)$. Here $L$ denotes the length of $\beta$ and $A \in(-\pi, \pi)$ the oriented area enclosed by $\beta$ in the two-sphere.

- The whole extrinsic geometry of $\mathbf{T}_{\beta}$ is governed by the curvature function of $\beta$ in $\mathbb{S}^{2}\left(\frac{1}{2}\right)$.

\subsection{A Useful Tool II: Lancret's Curves}

A general helix (or Lancret's curve) in $\mathbb{R}^{3}$ is a curve which makes a constant angle with a fixed straight line (the axis of the general helix). General helices can be characterized algebraically by means of a classical theorem: the ratio of torsion to curvature of a general helix is constant (M. Lancret 1802; B. de Saint Venant 1845). One has also a simple geometric characterization of these curves: A curve in $\mathbb{R}^{3}$ is a Lancret's curve, if and only if, it is a geodesic of a right cylinder shaped on a plane curve. Of course, ordinary helices, that is, curves with both constant curvature and constant torsion, are examples of general helices and they are called trivial Lancret's curves.

This notion has been extended to real space forms [14]: A unit speed curve $\gamma$ in $\mathbb{M}^{3}(G)$ will be called a general helix if there exists a Killing vector field $V(s)$ with constant length along $\gamma$ (the axis), such that the angle between $V$ and $\gamma^{\prime}$ is a non-zero constant along $\gamma$ [14].

This definition is the natural extension of that for general helices in $\mathbb{R}^{3}$ in which a straight line works as an axis. Obvious examples of general helices are "plane" curves, that is curves in $\mathbb{M}^{3}(G)$ with $\tau \equiv 0$ (just take $V=B$ to have an axis) and ordinary helices where $V(s)=\cos \theta \cdot T(s)+\sin \theta \cdot B(s)$ with $\cot \theta=\frac{\tau^{2}-G}{\tau \kappa}$ works as an axis. 
M. Barros proved the following theorem, which is an algebraic characterization of Lancret's curves in non-flat real three-space forms [14]

Proposition 1. (The Lancret theorem in three-space forms)

1) A curve $\gamma$ in the hyperbolic three-space $\mathbb{H}^{3}(-1)$ is a general helix, if and only if, either 1) $\tau \equiv 0$ and $\gamma$ is a curve in some hyperbolic plane, or 2) $\gamma$ is an ordinary helix.

2) A curve $\gamma$ in the unit three-sphere $\mathbb{S}^{3}(1)$ is a general helix, if and only if, either 1) $\tau \equiv 0$ and $\gamma$ is a curve in some unit two-sphere, or 2) there exists a constant $b$ such that

$$
\tau=b \kappa \pm 1
$$

As a consequence, only trivial examples of Lancret helices exist in $\mathbb{H}^{3}(-1)$. In contrast, there are plenty of non-trivial examples of Lancret curves in the unit three-sphere. These can be characterized geometrically by using the Hopf Cylinders in the following way [14]

Proposition 2. A curve $\gamma$ in $\mathbb{S}^{3}(1)$ is a general helix if and only if it is a geodesic of a Hopf cylinder. Moreover, $\gamma$ is an ordinary helix if and only if it is a geodesic of a Hopf cylinder shaped on a circle.

\section{Order one Functionals}

Now, we pay attention to order one energy functionals. First, we consider functionals belonging to the total curvature functional family. Thus, we are interested in the closed critical points of

$$
\mathcal{F}(\gamma)=\int_{\gamma}(\kappa+\lambda) \mathrm{d} s
$$

acting on an $n$-dimensional Riemannian manifold $\mathbb{M}^{n}$. If $\lambda=0$ we will say that (4) corresponds to the free model, while if $\lambda \neq 0$, we shall talk about the constrained model.

The Euler-Lagrange equations [4] associated to (4) are

$$
R(N, T) T=\left(\tau^{2}+\lambda \kappa\right) N-\tau_{s} B+\tau \Upsilon
$$


where $\Upsilon$ belongs to the vector bundle on the curve which is orthogonal to the bundle spanned by the Frenet frame, and the subscript $s$ means derivative with respect to the arclength parameter $s$.

As for the solutions to the free model $(\lambda=0)$ they have been studied in [4] and [5]. It has been proved there that the Gaussian curvature vanishes on critical points $\gamma$ lying on surfaces. Moreover, in a real space form $\mathbb{M}^{n}(G)$, trajectories actually lie in $\mathbb{M}^{3}(G)$, and, if $\gamma$ is a critical point for $\mathcal{F}$ which is fully immersed in $\mathbb{M}^{3}(G)$, then, $\tau^{2}=G>0$ ( $\tau$ represents the torsion of the curve). Therefore, up to rigid motions, non-trivial examples of critical points for $\mathcal{F}$, which are not contained in totally geodesic surfaces, can only occur in $\mathbb{S}^{3}(1)$. They can be characterized as horizontal lifts via the Hopf map of curves in $\left.\mathbb{S}^{2}\left(\frac{1}{2}\right)\right)$. Among them the closed critical points are given by [4]

Proposition 3. Let $\beta$ be an immersed closed curve in $\mathbb{S}^{3}(1)$. Then $\beta$ is a critical point for $\mathcal{F}$, if and only if, there exists a natural number, say $m$, such that $\beta$ is a horizontal lift, via the Hopf map, of the $m$-fold cover of an immersed closed curve $\gamma$ in $\mathbb{S}^{2}\left(\frac{1}{2}\right)$ whose enclosed oriented area $A$ is a rational multiple of $\pi, A=\frac{p}{m} \pi$, being $p$ and $m$ relative primes.

This proposition gives us an algorithm to construct many examples of closed critical points of the total mean curvature energy in $\mathbb{S}^{3}(1)$.

Example 4. The spherical elliptic lemniscate. Consider the curve which in spherical coordinates $(\phi, \theta)$ on $\mathbb{S}^{2}\left(\frac{1}{2}\right)$ is described by

$$
\gamma: \frac{1}{4}\left(\phi^{2}+\sin ^{2} \theta\right)^{2}=a^{2} \sin ^{2} \theta+b^{2} \phi^{2}
$$

with the parameters $a$ and $b$ satisfying $b^{2} \geq 2 a^{2}$.

This curve is the image under a Lambert projection of an elliptic lemniscate in the plane. Since the Lambert projection preserves the area, the area enclosed by $\gamma$ in $\mathbb{S}^{2}\left(\frac{1}{2}\right)$ is $A=\frac{a^{2}+b^{2}}{2} \pi$. Now we choose $a$ and $b$ such that $a^{2}+b^{2}$ is a rational number, say $\frac{p}{q}$, with $a^{2}+b^{2} \leq 1$. Then, a horizontal lift of the $2 q$-fold cover of $\gamma$ gives a closed critical point for $\mathcal{F}$ in $\mathbb{S}^{3}(1)$.

Example 5. The spherical limaçon or the spherical snail of Pascal. Given real parameters $a$ and $h$, we define the following curve of $\mathbb{S}^{2}\left(\frac{1}{2}\right)$

$$
\gamma:\left(\frac{1}{2} \phi^{2}+\frac{1}{2} \sin ^{2} \theta-2 a \phi\right)^{2}=h^{2}\left(\phi^{2}+\sin ^{2} \theta\right) .
$$


This is nothing but the image under the Lambert projection of a plane snail of Pascal. Therefore, the area enclosed by $\gamma$ is given by $A=\left(h^{2}+\frac{1}{2} a^{2}\right) \pi$. Again, for a suitable choice of parameters $a$ and $h$, we get examples of closed critical points for $\mathcal{F}$ in $\mathbb{S}^{3}(1)$ by applying the above proposition.

Similar arguments can be used to study the constrained model

$$
\mathcal{F}(\gamma)=\int_{\gamma}(\kappa+\lambda) \mathrm{d} s, \quad \lambda \neq 0 .
$$

In this case, the whole space of closed trajectories in the constrained model is formed by a rational one-parameter family of closed helices in $\mathbb{S}^{3}(1)$. Geometrically, they are geodesics of circular Hopf tori which are obtained when the slope is quantized by a rational constraint. Hence, the solution to the problem is encoded in the geometry of the Hopf Tori.

As we shall show in the last section, the above funcionals are of interest in Physics. And so are related energy functionals which are also of first order, not only in the curvature of the curve, but also in the other Frenet curvatures. They are given by

$$
\mathcal{F}_{\text {mnp }}(\gamma)=\int_{\gamma}(m+n \kappa+p \tau) \mathrm{d} s
$$

with $m, n, p \in \mathbb{R}$. This time, instead of studying closed trajectories, we assume that certain second order boundary conditions are satisfied. More concretely, given $q_{1}, q_{2} \in \mathbb{M}^{3}(G)$ and $\left\{x_{1}, y_{1}\right\},\left\{x_{1}, y_{1}\right\}$ orthonormal vectors in $T_{q_{1}} \mathbb{M}^{3}(G)$ and $T_{q_{2}} \mathbb{M}^{3}(G)$ respectively, we define the following space of curves

$$
\Lambda=\left\{\gamma:\left[t_{1}, t_{2}\right] \rightarrow \mathbb{M}^{3}(G) ; \gamma\left(t_{i}\right)=q_{i}, \quad T\left(t_{i}\right)=x_{i}, \quad N\left(t_{i}\right)=y_{i}, \quad 1 \leq i \leq 2\right\}
$$

Then, the critical points of the variational problem $\mathcal{F}_{m n p}: \Lambda \rightarrow \mathbb{R}$ are characterized by the following Euler-Lagrange equations (for details, see [7])

$$
-m \kappa+p \kappa \tau-n \tau^{2}+n c=0, \quad p \kappa_{s}-n \tau_{s}=0 .
$$

A direct analysis of this equation gives that the solutions to the problem can be classified according to the tables shown bellow.

From Table 1, we observe that, in the Euclidean space, non-trivial Lancret curves appear just for models with $m=0$ and $p . n \neq 0$, that is for energies of the type $\mathcal{F}_{0 n p}(\gamma)=\int_{\gamma}(n \kappa+p \tau) \mathrm{d} s$. In these cases the ratio $\frac{p}{n}$ determines the slope of the solutions. In other words, $\frac{p}{n}=\cot \theta$, where $\theta$ is the angle that the Lancret curve makes with the axis. 
Table 1. Critical points in $\mathbb{R}^{3}$

\begin{tabular}{|l|l|l|l|}
\hline$m$ & $n$ & $p$ & Solutions in $\mathbb{R}^{3}, G=0$ \\
\hline$\neq 0$ & $=0$ & $=0$ & Geodesics $\kappa=0$ \\
$=0$ & $=0$ & $\neq 0$ & Circles $\kappa$ constant and $\tau=0$ \\
$=0$ & $\neq 0$ & $=0$ & Plane curves $\tau=0$ \\
$\neq 0$ & $\neq 0$ & $=0$ & Ordinary Helices with $\kappa=\frac{-n \tau^{2}}{m}$ \\
$\neq 0$ & $=0$ & $\neq 0$ & Ordinary Helices with arbitrary $\kappa$ and $\tau=\frac{m}{p}$ \\
$=0$ & $\neq 0$ & $\neq 0$ & Lancret curves with $\tau=\frac{p}{n} \kappa$ \\
$\neq 0$ & $\neq 0$ & $\neq 0$ & Ordinary Helices with $\kappa=\frac{-n a^{2}}{m+a p}, \tau=\frac{m a}{m+a p}$ and \\
& & & $a \in \mathbb{R}-\left\{-\frac{m}{p}\right\}$ \\
\hline
\end{tabular}

Table 2. Critical points in $\mathbb{H}^{3}(G)$

\begin{tabular}{|l|l|l|l|}
\hline$m$ & $n$ & $p$ & Solutions in $\mathbb{H}^{3}(G), G=-c^{2}$ \\
\hline$\neq 0$ & $=0$ & $=0$ & Geodesics $\kappa=0$ \\
$=0$ & $=0$ & $\neq 0$ & Curves with $\kappa$ constant and $\tau=0$ \\
$=0$ & $\neq 0$ & $=0$ & Do not exist \\
$\neq 0$ & $\neq 0$ & $=0$ & Ordinary Helices with $\kappa=\frac{-n\left(c^{2}+\tau^{2}\right)}{m}$ \\
$\neq 0$ & $=0$ & $\neq 0$ & Ordinary Helices with arbitrary $\kappa$ and $\tau=\frac{m}{p}$ \\
$=0$ & $\neq 0$ & $\neq 0$ & Ordinary Helices with $\kappa=\frac{-n\left(c^{2}+a^{2}\right)}{a p} \tau=-\frac{c^{2}}{a}$ and \\
$\neq 0$ & $\neq 0$ & $\neq 0$ & $\begin{array}{l}a \in \mathbb{R}-\{0\} \\
\text { Ordinary Helices with } \kappa=\frac{-n\left(c^{2}+a^{2}\right)}{m+a p}, \tau=\frac{m a-p c^{2}}{m+a p} \text { and }\end{array}$ \\
& & & $a \in \mathbb{R}-\left\{-\frac{m}{p}\right\}$ \\
\hline
\end{tabular}

As for the Hyperbolic space, only trivial examples can be obtained as Table 2 shows.

Again, the spherical case shows the richest behavior. By observing Table 3 one sees that the most interesting models on spheres are those where m.n.p $\neq 0$. Remember that general helices in $\mathbb{S}^{3}(1)$ are completely determined from both a curve in the $\mathbb{S}^{2}\left(\frac{1}{2}\right)$ and the slope, that is the angle that the helix makes, in the corresponding Hopf tube, with the axis (i.e., with the fibres). In these cases the ratio $\frac{m}{p}$ is determined from the radius of the sphere while the ratio $\frac{p}{n}$ gives the slope of the solutions. Notice that, in particular, the horizontal lifts of curves in the two sphere are general helices of the three sphere with slope $\frac{\pi}{2}$. Now, let $\beta_{n p}$ denote the geodesic in $\mathbf{T}_{\beta}=\pi^{-1}(\beta)$ with slope $\theta, \cot \theta=\frac{p}{n}$. From the third table one has, for example, the following 
Proposition 6. Let $\gamma$ be a curve in $\mathbb{S}^{3}(1)$, then it is a critical point of $\mathcal{F}_{n n p}, n . p \neq$ 0 , if and only if either 1) $\gamma$ is a helix with curvature $\kappa=\frac{n\left(1-a^{2}\right)}{n+a p}$ and torsion $\tau=\frac{n a+p}{n+a p}$ where $a \in \mathbb{R}-\left\{-\frac{n}{p}\right\}$, or 2) $\gamma \in\left\{\beta_{n p} ; \beta\right.$ is a curve in $\left.\mathbb{S}^{2}\left(\frac{1}{2}\right)\right\}$.

Table 3. Critical points in $\mathbb{S}^{3}(G)$

\begin{tabular}{|l|l|l|l|}
\hline$m$ & $n$ & $p$ & Solutions in $\mathbb{S}^{3}(G), G=c^{2}$ \\
\hline$\neq 0$ & $=0$ & $=0$ & Geodesics $\kappa=0$ \\
$=0$ & $=0$ & $\neq 0$ & Circles $\kappa$ constant and $\tau=0$ \\
$=0$ & $\neq 0$ & $=0$ & Horizontal lifts, via the Hopf map, of curves in $\mathbb{S}^{2}$ \\
$\neq 0$ & $\neq 0$ & $=0$ & Ordinary Helices with $\kappa=\frac{n\left(c^{2}-\tau^{2}\right)}{m}$ \\
$\neq 0$ & $=0$ & $\neq 0$ & Ordinary Helices with arbitrary $\kappa$ and $\tau=\frac{m}{p}$ \\
$=0$ & $\neq 0$ & $\neq 0$ & Ordinary Helices with $\kappa=\frac{n\left(c^{2}-a^{2}\right)}{a p}, \tau=\frac{c^{2}}{a}$ and \\
$\neq 0$ & $\neq 0$ & $\neq 0$ & $\begin{array}{l}\text { Ordinary Helices with } \kappa=\frac{n\left(c^{2}-a^{2}\right)}{m+a p}, \tau=\frac{m a+p c^{2}}{m+a p} \text { and } \\
\neq 0\end{array}$
\end{tabular}

Now, if we again restrict ourselves to the variational problem on the space of closed curves, one can show that, other than closed "plane" curves (zero torsion), there are no closed critical points either in $\mathbb{R}^{3}$ or in $\mathbb{H}^{3}(G)$. As for the unit sphere, closed general helices in $\mathbb{S}^{3}(1)$ can be characterized with the aid of Table 3 . Since, we are in the unit sphere, in order to find critical points with non-constant Frenet curvatures, we must take $n \neq 0, m=p \neq 0$. For any curve $\beta(s)$ in $\mathbb{S}^{2}\left(\frac{1}{2}\right)$, take $\mathbf{T}_{\beta}=\pi^{-1}(\beta)$ the Hopf Cylinder shaped on $\beta$. Then, from the isometry type of $\mathbf{T}_{\beta}$, we have that a geodesic $\gamma$ of $\mathbf{T}_{\beta}$ closes up, if and only if, its slope $\omega=\cot \theta$ satisfies

$$
\omega=\frac{1}{L}(2 A+q \pi)
$$

where $q$ is a rational number. Finally, $\gamma \in \Omega$ is a critical point of $\mathcal{F}_{p n p}$ if and only if its slope satisfies $\omega=\frac{p}{n}$. Then, we have

Proposition 7. Let $\beta$ be an embedded closed curve in $\mathbb{S}^{2}\left(\frac{1}{2}\right)$, with length $L>0$ and enclosing an oriented area $A \in(-\pi, \pi)$. Then, the geodesic with slope $\omega$ in $\mathbf{T}_{\beta}=\pi^{-1}(\beta)$ is a critical point of the variational problem $\mathcal{F}_{p n p}: \Omega \rightarrow \mathbb{R}$ in $\mathbb{S}^{3}(1)$ if and only if the following relationship holds

$$
\frac{\omega L-2 A}{\pi} \in \mathbb{Q}
$$


We can assume the area $A$ to be positive, changing if necessary the orientation of $\beta$. The only further restriction on $(A, L)$ to define an embedded closed curve in the two sphere is given by the isoperimetric inequality in $\mathbb{S}^{2}\left(\frac{1}{2}\right)$

$$
L^{2}+4 A^{2}-4 \pi A \geq 0 .
$$

In terms of $(2 A, L)$, the above inequality can be written as $L^{2}+(2 A-\pi)^{2} \geq \pi^{2}$. If we define in the $(2 A, L)$-plane the region

$$
\Delta=\left\{(2 A, L) ; L^{2}+(2 A-\pi)^{2} \geq \pi^{2} \quad \text { and } \quad 0 \leq A \leq \pi\right\}
$$

then, we have that for each point $(2 A, L) \in \Delta$ there is an embedded closed curve on $\mathbb{S}^{2}\left(\frac{1}{2}\right)$ with length $L$ and enclosed area $A$. Therefore, one can obtain

Theorem 8. For any couple of parameters, $n$ and $p$ with $n . p \neq 0$, there exists an infinite series of closed general helices that are extremal for the variational problem $\mathcal{F}_{\text {pnp }}: \Omega \rightarrow \mathbb{R}$ in $\mathbb{S}^{3}(1)$. This series includes all the geodesics $\beta_{n p}$ in $\mathbf{T}_{\beta}=\pi^{-1}(\beta)$ with slope $\omega=\frac{p}{n}$ and where $\beta$ is determined as above by a pair $(2 A, L)$ belonging to the following region

$$
\Delta \cap\left(\cup_{q \in \mathbb{Q}}(\omega L-2 A=q \pi)\right) .
$$

\section{Higher Order Functionals}

We consider now the variational problem associated to the generalized EulerBernoulli energy functional

$$
\mathcal{F}(\gamma)=\int_{\gamma} P(\kappa) \mathrm{d} s
$$

acting on $\mathcal{H}$, a certain space of curves lying in a Riemannian manifold $\mathbb{M}^{n}$ and verifying suitable boundary conditions, where now $P$ is a $C^{\infty}$ function satisfying $\frac{\mathrm{d} P^{\prime}}{\mathrm{d} s} \neq 0$ (here $P^{\prime}(\kappa)=\frac{\mathrm{d} P}{\mathrm{~d} \kappa}$ ) and $s$ is the curve parameter. We follow a method mainly due to J. Langer and D. Singer [38]. The next result was obtained in [38]

Lemma 9. With the notation introduced in $\S 2$ we have:

1. $[V, W]=0$.

2. $[W, T]=g T$, where $\left\langle\nabla_{T} W, T\right\rangle=-g$

3. $[[W, T], T]=-T(g) T=-g_{s} T$ 

4. $\frac{\partial v}{\partial w}=\left\langle\nabla_{T} W, T\right\rangle v=-g v$
5. $\frac{\partial \kappa}{\partial w}=\left\langle R(W, T) T, \nabla_{T} T\right\rangle+\left\langle\nabla_{T}^{2} W, N\right\rangle-2\left\langle\nabla_{T} W, T\right\rangle \kappa$

Moreover, if $\mathbb{M}^{n}(G)$ has constant sectional curvature $G$ then

$$
\frac{\partial \tau}{\partial w}=\left\langle\frac{1}{\kappa} \nabla_{T}^{3} W-\frac{\kappa_{s}}{\kappa^{2}} \nabla_{T}^{2} W, B\right\rangle+\left(\frac{G}{\kappa}+\kappa\right) \nabla_{T} W-\frac{\kappa_{s}}{\kappa^{2}}\langle G W, B\rangle .
$$

We define the following vector fields

$$
\begin{aligned}
\mathcal{K} & =P^{\prime}(\kappa) \cdot N \\
\mathcal{J} & =\nabla_{T} \mathcal{K}+\left(2 \kappa P^{\prime}(\kappa)-P(\kappa)\right) \cdot T \\
\mathcal{E} & =\nabla_{T} \mathcal{J}+P^{\prime}(\kappa) \cdot R(N, T) T .
\end{aligned}
$$

Then, by using Lemma 9, the first Frenet formula $\nabla_{T} T=\kappa N$ and integration by parts, we can obtain the first derivative of $\mathcal{F}$ [2]

Proposition 10. (First Variation Formula) Under the above conditions and notation, the following formula holds

$$
\frac{\mathrm{d}}{\mathrm{d} w} \mathcal{F}(\gamma)_{\mid w=o}=\int_{0}^{L}\langle\mathcal{E}, W\rangle \mathrm{d} s+\mathcal{B}[W, \gamma]_{0}^{L}
$$

where

$$
\mathcal{B}[W, \gamma]_{0}^{L}=\left[\left\langle\mathcal{K}, \nabla_{T} W\right\rangle-\langle\mathcal{J}, W\rangle\right]_{0}^{L}
$$

is the boundary operator.

Thus, under suitable boundary conditions, one sees that a critical point of $\mathcal{F}$ will satisfy the following Euler-Lagrange equation

$$
\mathcal{E}=\nabla_{T}^{2} P^{\prime}(\kappa) \cdot N+\nabla_{T}\left(2 \kappa P^{\prime}(\kappa)-P(\kappa)\right) \cdot T+P^{\prime}(\kappa) \cdot R(N, T) T=0 .
$$

When the manifold $\mathbb{M}^{n}(G)$ is a real space form of constant curvature $G$ the equation (12) reduces to

Proposition 11. (Euler-Lagrange equations in real space forms)

$$
\begin{aligned}
\left(\kappa^{2}-\tau^{2}+G\right) P^{\prime}(\kappa)+\frac{\mathrm{d}^{2} P^{\prime}(\kappa)}{\mathrm{d} s^{2}} & =\kappa P(\kappa) \\
2 \frac{\mathrm{d} P^{\prime}(\kappa)}{\mathrm{d} s} \tau+P^{\prime}(\kappa) \tau_{s} & =0 \\
P^{\prime}(\kappa) \eta & =0 .
\end{aligned}
$$


Here $\kappa_{s}, \tau_{s}$ denote derivative with respect to the arclength parameter and $\eta$ belongs to the normal bundle to span $\{T, N, B\}$.

A consequence of (15) is that a critical point $\gamma$ must fully lie in either a twodimensional or a three-dimensional totally geodesic submanifold of $\mathbb{M}^{n}(G)$. Thus, our problem in space forms reduces to explicitly determine the closed critical curves in a manifold whose dimension is two and three.

Solving the Euler-Lagrange equations is not an easy task even in real space forms. Integration of $\mathcal{E}=0$ in $\mathbb{M}^{3}(G)$ is all but impossible for a general $P$. Even if we assume the existence of periodic solutions $\kappa, \tau$, the corresponding periodic curves in $\mathbb{M}^{3}(G)$ are not necessarily closed. Then, we need to establish conditions for these critical points to close up and to calculate the second variation formula to locate minima. For a general $P$ we know how to the compute first integrals of $\mathcal{E}=0$ and give closedness conditions for the critical curves. We also can compute the second variation formula [10]. Moreover, for "suitable" choices of $P$, we additionally know how to solve the Euler-Lagrange equations (explicitly or by quadratures) and determine the closed critical points.

First, we integrate the Euler-Lagrange equations. The idea consists in the use of a family of Killing fields along a critical point $\gamma(s)$ which are expressible in terms of the local invariants of the curve, in combination with a sort of Noether's argument to facilitate the integration. From (3) and Lemma 9 one has that $W$ is a Killing field along $\gamma$, if and only if,

$$
\begin{aligned}
\left\langle\nabla_{T} W, T\right\rangle & =0 \\
\left\langle\nabla_{T}^{2} W, N\right\rangle+G \cdot\langle W, N\rangle & =0 \\
\left\langle\frac{1}{\kappa} \nabla_{T}^{3} W-\frac{\kappa_{s}}{\kappa^{2}} \nabla_{T}^{2} W+\left(\frac{G}{\kappa}+\kappa\right) \nabla_{T} W-\frac{\kappa_{s}}{\kappa^{2}} G \cdot W, B\right\rangle & =0 .
\end{aligned}
$$

Then, with the aid of formulas (16)-(18), it is possible to show that the vector fields $\mathcal{J}$ and $\mathcal{I}$ defined by

$$
\begin{aligned}
\left(\kappa P^{\prime}(\kappa)-P(\kappa)\right) \cdot T+\frac{\mathrm{d} P^{\prime}(\kappa)}{\mathrm{d} \kappa} \cdot N+\tau P^{\prime}(\kappa) \cdot B & =\mathcal{J} \\
-P^{\prime}(\kappa) \cdot B & =\mathcal{I}
\end{aligned}
$$

are Killing vector fields along $\gamma$.

Proposition 12. Let $\gamma: \mathbb{I}=[0,1] \rightarrow \mathbb{M}^{3}(G)$ be a critical point of $\mathcal{F}$. Then the vector fields $\mathcal{J}$ and $\mathcal{I}$ defined in (19) and (20) are Killing fields along $\gamma$. 
Now if $\gamma$ happens to be a critical point of $\mathcal{F}$ (under any boundary conditions), then standard arguments imply that $\mathcal{E}=0$ on $\gamma$. The variation formulas continue to hold when $L$ is replaced by any intermediate value $t \in(0, L)$ and, therefore, the first variational formula (proposition 10) reduces to

$$
\frac{\mathrm{d}}{\mathrm{d} w} \mathcal{F}(\gamma)_{\left.\right|_{w=o}}=\mathcal{B}[W, \gamma]_{0}^{t}
$$

Therefore, for any Killing field $W$ on $\mathbb{M}^{3}(G)$, we have from (21)

$$
0=\mathcal{B}[W, \gamma]_{0}^{t}
$$

Thus, $\mathcal{B}[W, \gamma](t)$ is constant along $\gamma$. Applying this to $\mathcal{I}, \mathcal{J}$, we have

$$
\begin{aligned}
\langle\mathcal{I}, \mathcal{J}\rangle & =c \\
\langle\mathcal{I}, \mathcal{J}\rangle+G\langle\mathcal{I}, \mathcal{I}\rangle & =e
\end{aligned}
$$

on $\gamma$, where $c$ and $e$ are constants. Now, plug (19) and (20) into (23) and (24) to obtain

Proposition 13. (First Integrals of the Euler-Lagrange equations in space forms) With the above notation,

$$
\begin{array}{r}
\tau \cdot\left(P^{\prime}(\kappa)\right)^{2}=e \\
\left(P^{\prime \prime}(\kappa)\right)^{2} \cdot \kappa_{s}^{2}+\left(\kappa \cdot P^{\prime}(\kappa)-P(\kappa)\right)^{2}+G \cdot\left(P^{\prime}(\kappa)\right)^{2}+\frac{e^{2}}{\left(P^{\prime}(\kappa)\right)^{2}}=d
\end{array}
$$

for some constants $e, d \in \mathbb{R}$.

In order to establish closedness conditions for the critical points $\gamma$ associated to periodic solutions of the Euler-Lagrange equation, one can construct an adapted coordinate system which depends on both the space of Killing fields of $\mathbb{M}^{3}(G)$ and on the choice of $P$. Assume that $\kappa(s), \tau(s)$ are periodic solutions of EulerLagrange equations. They determine a unique (up to rigid motions) curve $\gamma(s)$ in $\mathbb{M}^{3}(G)$. Denote by $\mathcal{J}, \mathcal{I}$ both the associated Killing fields along $\gamma(s)$ defined in (20) (19) and their extensions to $\mathbb{M}^{3}(G)$. Then, we have the following [11]

Proposition 14. The Killing fields $\mathcal{J}, \mathcal{I}$ commute, i.e., $[\mathcal{J}, \mathcal{I}]=0$.

The above result can be used to find a coordinate system where the coordinates of $\gamma(s)$ and its closedness conditions can be expressed in terms of $P$ and $\kappa$. For 
instance, in order to establish the closedness conditions in $\mathbb{S}^{3}(1)$, we can choose cylindrical coordinates in the three-sphere

$$
x(\theta, \varphi, \psi)=(\cos \theta \cos \psi, \sin \theta \cos \psi, \cos \varphi \sin \psi, \sin \varphi \sin \psi)
$$

with $\theta, \varphi \in(0,2 \pi), \psi \in\left(0, \frac{\pi}{2}\right)$, where $\gamma(s)$ is given by

$$
\gamma(s)=x(\theta(s), \varphi(s), \psi(s)) .
$$

By combining Proposition 14, the expressions for $\mathcal{J}$ and $\mathcal{I}$ (19), (20), and the first integrals of $\mathcal{E}=0,(23),(24)$, one can obtain

$$
\begin{aligned}
\theta^{\prime}(s) & =\frac{b\left(\kappa P^{\prime}(\kappa)-P(\kappa)\right)}{b^{2}-\left(P^{\prime}(\kappa)\right)^{2}} \\
\varphi^{\prime}(s) & =\frac{a\left(\kappa P^{\prime}(\kappa)-P(\kappa)\right)}{a^{2}-\left(P^{\prime}(\kappa)\right)^{2}} \\
\cos 2 \psi & =2 \frac{\left(P^{\prime}(\kappa)\right)^{2}-b^{2}}{a^{2}-b^{2}}-1
\end{aligned}
$$

for certain constants $a, b \in \mathbb{R}$. Hence, from the set of equations (28) we have that the curvature $\kappa$ and the energy function $P$ basically determine the cylindrical coordinates $\theta(s), \varphi(s), \psi(s)$ of a critical point $\gamma(s)$. Moreover, closedness conditions for the critical point $\gamma(s)$ can be formulated in this system in the following way [2], [11]

Proposition 15. Let $\gamma$ be a curve in $\mathbb{S}^{3}(1)$ which is a critical point of the energy (7) with periodic curvature. Then, $\gamma$ will close up, if and only if, the angular progressions

$$
\begin{aligned}
& \Lambda_{\theta}(\gamma)=\int_{o}^{\rho} \frac{b\left(\kappa P^{\prime}(\kappa)-P(\kappa)\right)}{b^{2}-\left(P^{\prime}(\kappa)\right)^{2}} \mathrm{~d} s \\
& \Lambda_{\varphi}(\gamma)=\int_{o}^{\rho} \frac{a\left(\kappa P^{\prime}(\kappa)-P(\kappa)\right)}{a^{2}-\left(P^{\prime}(\kappa)\right)^{2}} \mathrm{~d} s
\end{aligned}
$$

are rational multiples of $2 \pi$.

By using similar arguments, one can use an adapted cylindrical coordinate system $r(s), z(s), \varphi(s)$ in $\mathbb{R}^{3}$ to show that the coordinates of a critical curve $\gamma(s)$ can also 
be expressed in terms of $\kappa(s)$ and $P(\kappa)$. A critical point with periodic curvature $\gamma$ will close up in $\mathbb{R}^{3}$, if and only if [2]

$$
0=\int_{o}^{\rho}\left(\kappa P^{\prime}(\kappa)-P(\kappa)\right) \mathrm{d} s
$$

and the angular progression

$$
\Lambda_{\varphi}(\gamma)=\int_{o}^{\rho} \frac{e \sqrt{d}\left(\kappa P^{\prime}(\kappa)-P(\kappa)\right)}{e^{2}-d\left(P^{\prime}(\kappa)\right)^{2}} \mathrm{~d} s
$$

is a rational multiple of $2 \pi(e, d \in \mathbb{R})$.

The two-dimensional versions for the closedness conditions in $\mathbb{R}^{2}$ and $\mathbb{S}^{2}(1)$ are obtained by taking $b=0$ and $e=0$ in the above formulas. Analogously, closedness conditions in $\mathbb{H}^{2}$ can also be stated [2].

The second variation formula can be computed also for a general $P$ [2], although it is so complicated that, if one wants to go any further, we need to consider suitable particular choices of $P$, where by "suitable" we mean that the Euler-Lagrange equations $\mathcal{E}=0$ can be explicitly solved (or, at least, they can be solved by quadratures) and/or that $P(\kappa)$ has mathematical and/or physical significance. Examples of suitable choices where the method works include the family of energy functionals of the type $P(\kappa)=(\kappa+\lambda)^{r}$. In particular, for the energy functionals shown below a significant advance has been made

$$
P(\kappa)=\kappa^{r} \text { (hyperelastic curves) }\left\{\begin{array}{l}
r=1 \text { (total curvature functional) } \\
r=2 \text { (classical elasticae functional) } \\
r>2 \text { (proper hyperelasticae functional) }
\end{array}\right.
$$

$$
\begin{aligned}
& P(\kappa)=(\kappa+\lambda)^{2} \quad(\text { elasticae circular at rest }) \\
& P(\kappa)=(\kappa+\lambda)^{\frac{1}{2}} \quad(\text { constrained total curvature })
\end{aligned}
$$

The variational problem associated to these energies has been considered for many authors. For instance, the total mean curvature energy has been studied in twodimensional and three-dimensional real space forms in [4], [5]. We have given a short review of that in Section three. As we have mentioned, the classical elasticae 
problem was first analyzed by L. Euler and D. Bernoulli. On the other hand, J. Langer and D. Singer [38] classified the closed elasticae in two-dimensional real space forms. They also classified closed elasticae in $\mathbb{R}^{3}$ [39]. Closed helical elasticae in $\mathbb{S}^{3}(1)$ were determined in [11]. There exist no closed proper hyperelasticae $(r>2)$ in $\mathbb{R}^{2}, \mathbb{S}^{2}(1)$ and $\mathbb{R}^{3}[10]$. Closed $r$-elasticae in $\mathbb{H}^{2}(-1)$ have been studied in [6], [8]. In particular, if $r=3$, they provide nice applications to the study of natural extension of the Nambu-Goto-Poliakov action and to the determination of the first examples of Chen-Willmore hypersurfaces in $\mathbb{R}^{4}$ [6]. Closed helical $r$-elasticae in $\mathbb{S}^{3}(1)$ were determined in [11]. Closed elasticae circular at rest are determined in $\mathbb{S}^{2}(1)$ and $\mathbb{H}^{2}(-1)$ [12], [13]. In [11] we studied also closed critical curves for the constrained total curvature in $\mathbb{S}^{2}(1)$. As we shall show in the last section, these results have applications to the study of Willmore surfaces, vesicles and membranes, p-branes and string theory, Chen-Willmore submanifolds, etc... But now, let us discuss shortly some of the previous functionals.

\section{Natural Choices of the Energies}

We are going to extend further our analysis for two concrete examples of "suitable" choices of the Lagrangian $P$.

\subsection{Classical Elasticae in $\mathbb{S}^{3}(1)$}

We wish to study the critical points of the elastic energy functional

$$
\mathcal{F}(\gamma)=\int_{\gamma} \kappa^{2} \mathrm{~d} s
$$

acting on closed curves of the three-sphere (for details, see [11]). First, we investigate the critical points with constant curvature. From (25), it turns out that they also must have constant non-zero torsion and, therefore, they are ordinary helices. Moreover, we can prove that the set of constant curvature closed critical curves of $\mathcal{F}(\gamma)=\int_{\gamma} \kappa^{2} \mathrm{~d}$ s in $\mathbb{S}^{3}(1)$ is completely determined and forms a rational one-parameter family $\left\{\gamma_{q} ; q \in \mathbb{Q}^{+}-\left\{\frac{1}{2}\right\}\right\}$ [11]. The main point of the proof is that helices in $\mathbb{S}^{3}(1)$ can be considered as geodesics of Hopf tori and, as it has been pointed out in Sections 2 and 3, given a helix of known curvature and torsion $(\kappa, \tau)$, it may be seen as the geodesic of slope $g=\frac{1-\tau}{\kappa}$ contained in the Hopf torus $\mathbf{T}_{\alpha}$ shaped on the circle $\alpha$ of curvature $\rho=\frac{\kappa^{2}+\tau^{2}-1}{\rho}$ and enclosing an 
oriented area $A$ of the sphere $\mathbb{S}^{2}\left(\frac{1}{2}\right)$. Moreover, $\mathbf{T}_{\alpha}$ is determined by the lattice $\Gamma=\operatorname{span}\{(0,2 \pi),(L, 2 A)\}$, where $L$ is the length of $\alpha$. Hence, a helix will be closed, if and only if, there exists a rational number $q \neq 0$, such that

$$
g=q \sqrt{\rho^{2}+4}-\frac{\rho}{2}
$$

Thus, given $\rho \in \mathbb{R}, q \in \mathbb{Q}$ we determine $g$ by (30). Then, the curvature and torsion $(\kappa, \tau)$ of the closed helix are obtained from $g=\frac{1-\tau}{\kappa}, \rho=\frac{\kappa^{2}+\tau^{2}-1}{\rho}$. Now, in order the helix to be a critical point, it must satisfy the Euler-Lagrange equations $\mathcal{E}=0$ (13), (14). In our case, this means that we must find a real number $\rho$ and a rational number $q$ satisfying $\mathcal{E}(\kappa(\rho, q), \tau(\rho, q))=0$. We can show that, for any rational number $q \neq 0$, there exists a unique positive solution obtaining in this way closed helical elasticae in $\mathbb{S}^{3}(1)$.

Secondly, we look for closed elasticae in $\mathbb{S}^{3}(1)$ with non-constant curvature function $\kappa$. In order to determine the closed critical points, the method requires to explicitly obtain the periodic solutions $\kappa, \tau$, of the Euler-Lagrange equations and then we need to compute the ingredients in the closedness conditions. Finally we should check that the closedness conditions are satisfied.

By applying Proposition 13, the first integrals of the Euler-Lagrange equations in this case are

$$
\begin{aligned}
4 d \kappa^{2}-16 G \kappa^{4}-4 \kappa^{6}-e^{2} & =16 \kappa^{2} \kappa_{s}^{2}(s) \\
\left(\frac{e}{4 \kappa^{2}(s)}\right) & =\tau(s)
\end{aligned}
$$

where $d$ and $e$ are constants of integration. The family of periodic solutions of these equations can be parameterized in

$$
\mathcal{D}=\left\{(\beta, \alpha) \in \mathbb{R}^{2} ; \alpha>\beta>0\right\}
$$

where $\beta, \alpha$ are determined by

$$
e^{2}=4(4 G+\alpha+\beta) \alpha \beta, \quad d=(\alpha+\beta)(4 G+\alpha+\beta)-\alpha \beta .
$$

This family is given by

$$
\kappa_{\beta, \alpha}^{2}(s)=\alpha-(\alpha-\beta) \operatorname{sn}^{2}\left(\frac{\sqrt{\alpha-\alpha_{o}}}{2} s-K(p), p\right)
$$

with $K(p)$ denoting the complete elliptic integral of the first kind of modulus $p=\sqrt{\frac{\alpha-\beta}{\alpha-\alpha_{o}}}$. Once we know the curvature, the torsion of the critical point can be obtained from (32). Therefore, we have proved 
Proposition 16. There exists a two-parameter family of curves in $\mathbb{S}^{3}(1), \mathcal{R}_{\beta, \alpha}=$ $\left\{\gamma_{\beta, \alpha} ; \alpha>\beta>0\right\}$, whose curvature and torsion functions $\kappa_{\beta, \alpha}$ and $\tau_{\beta, \alpha}$ are periodic solutions of the Euler-Lagrange equations corresponding to the elastic energy functional $\mathcal{F}$.

Members of $\mathcal{R}_{\beta, \alpha}$ are candidates to be closed critical points of $\mathcal{F}$. We must check, which among them (if they exist) satisfy the closedness conditions. Take $\gamma_{\beta, \alpha} \in \mathcal{R}_{\beta, \alpha}$ and let $\rho$ the period of its curvature $\kappa_{\beta, \alpha}(s)$. Then, according to Proposition $15, \gamma_{\beta, \alpha}$ is a closed critical point of $\mathcal{F}$, if and only if, the progression angles

$$
\begin{aligned}
& \Lambda \theta\left(\gamma_{\beta, \alpha}\right)=-\frac{b}{4} \int_{0}^{\rho(\beta, \alpha)}\left(\frac{\kappa^{2}}{\kappa^{2}-\frac{b^{2}}{4}}\right) \mathrm{d} s \\
& \Lambda \varphi\left(\gamma_{\beta, \alpha}\right)=-\frac{a}{4} \int_{0}^{\rho(\beta, \alpha)}\left(\frac{\kappa^{2}}{\kappa^{2}-\frac{a^{2}}{4}}\right) \mathrm{d} s
\end{aligned}
$$

are rationally related to $2 \pi$. To simplify the notation, we are using $\kappa$ instead of $\kappa_{\beta, \alpha}$ in the above formulas and $a$ and $b$ are given by

$$
a^{2}=\frac{d+\sqrt{d^{2}-4 e^{2}}}{2}, \quad b^{2}=\frac{d-\sqrt{d^{2}-4 e^{2}}}{2} .
$$

We define new parameters $(w, r)$ by $w=\frac{b^{2}}{4}$ and $r=\frac{a^{2}}{4}$. Then we can show after a long computation that

$$
\begin{aligned}
& \Lambda \theta(\gamma)=-\left(\frac{4 w}{\alpha-\alpha_{o}}\right)^{\frac{1}{2}}\left(K(p)+\frac{w}{\alpha-w} \Pi\left(\frac{\pi}{2}, \frac{\alpha-\beta}{\alpha-w}, \sqrt{\frac{\alpha-\beta}{\alpha-\alpha_{o}}}\right)\right) \\
& \Lambda \varphi(\gamma)=-\left(\frac{4 r}{\alpha-\alpha_{o}}\right)^{\frac{1}{2}}\left(K(p)+\frac{r}{\alpha-r} \Pi\left(\frac{\pi}{2}, \frac{\alpha-\beta}{\alpha-r}, \sqrt{\frac{\alpha-\beta}{\alpha-\alpha_{o}}}\right)\right)
\end{aligned}
$$

where we have written $\gamma=\gamma_{\beta, \alpha}$ for simplicity, and with $\Pi\left(\frac{\pi}{2}, v, p\right)$ (respectively, $K(p)$ ) representing the complete elliptic integral of third kind (respectively, of the first kind) of modulus $p=\sqrt{\frac{\alpha-\beta}{\alpha-\alpha_{o}}}$.

Now, for any $(\beta, \alpha) \in \mathcal{D}$, let $\kappa_{\beta, \alpha}(s)$ be the corresponding non-constant periodic solutions of the Euler-Lagrange equations. It determines a curve $\gamma_{\beta, \alpha}$ in $\mathbb{S}^{3}(1)$ belonging to $\mathcal{R}_{\beta, \alpha}$. Then, we use (33) and (34) to define the map

$$
\Lambda: \mathcal{D} \rightarrow \mathbb{R}^{2}
$$




$$
\Lambda(\beta, \alpha)=\left(\frac{\Lambda \varphi\left(\gamma_{\beta, \alpha}\right)}{2 \pi}, \frac{\Lambda \theta\left(\gamma_{\beta, \alpha}\right)}{2 \pi}\right) .
$$

To determine the closed critical curves of $\mathcal{R}_{\beta, \alpha}$, we must compute $\Lambda(\mathcal{D})$ as accurately as possible and show that $\Lambda(\mathcal{D}) \cap \mathbb{Q}^{2} \neq \varnothing$. In our case, we can prove that

$$
\Lambda(\mathcal{D})=\left\{(x, y) ; x^{2}+y^{2}<\frac{1}{2}, x>0 \text { and } y<-\frac{1}{2}\right\} .
$$

Hence, closed non-constant curvature elastic curves in $\mathbb{S}^{3}(1)$ are indexed in the set $\mathcal{A}=\Lambda(\mathcal{D}) \cap \mathbb{Q}^{2}$ (multiple covers of a closed elastica correspond to the same point of the region). Points in the upper boundary of the region $\mathcal{A}$ represent closed elastic curves that lie in $\mathbb{S}^{2}(1)$ (geodesics correspond to the "vertex" $\left(\frac{1}{2},-\frac{1}{2}\right)$ of $\mathcal{A})$, while points in the lower boundary of $\mathcal{A}$ correspond to closed elastic helices fully immersed in $\mathbb{S}^{3}(1)$. Finally, we have [11]

Proposition 17. For any choice of natural parameters $n, m, l \in \mathbb{N}$ satisfying

$$
(n, m, l)=1, \quad 0<m<\frac{n}{2}<l<\frac{n}{\sqrt{2}}, \quad m^{2}+l^{2}<\frac{n^{2}}{2}
$$

there exists a closed elastica $\gamma_{n, m, l}$ which is totally determined and which is fully immersed in $\mathbb{S}^{3}(1)$. Moreover, $\gamma_{n, m, l}$ closes up after $n$ periods of its curvature, $m$ trips around the "equator" associated to $x_{\varphi}$ in $\mathbb{S}^{3}(1)$, and l trips around the "equator" associated to $x_{\theta}$ in $\mathbb{S}^{3}(1)$.

Every closed elastica in $\mathbb{S}^{3}(1)$ can be obtained in this way.

\subsection{Elastic Curves Circular at Rest}

Our next example takes account of the variational problem connected with the closed elastic curves which are circular at rest, that is, with the critical points of

$$
\mathcal{F}^{\lambda}(\gamma)=\int_{\gamma}(\kappa-\lambda)^{2} \mathrm{~d} s
$$

in a surface of constant curvature $\mathbb{M}^{2}(c)$. For simplicity, sometimes critical curves of (35) will be called $\lambda$-elastic curves in $\mathbb{M}^{2}(c)$. As we shall see, they have not only intrinsic interest, but also they provide solutions to the membranes problem. We start with the two-sphere $\mathbb{S}^{2}(1)$. By using Propositions 11 and 13, critical points must satisfy the Euler-Lagrange equation

$$
2 \kappa_{s s}+\kappa^{3}+\left(2-\lambda^{2}\right) \kappa+2 \lambda=0
$$


whose first integral is

$$
4 \kappa_{s}^{2}=d-(\kappa+\lambda)^{2}\left((\kappa-\lambda)^{2}+4\right), \quad 0<d \in \mathbb{R} .
$$

The polynomial $Q_{d}(x)=d-(x+\lambda)^{2}\left((x-\lambda)^{2}+4\right)$ may have two or four simple roots depending on the values of $\lambda$ and $d$. If it has four roots, then for any $d$ we obtain two solutions of (36). The first one is given by

$$
\kappa_{d}^{\lambda}(s)=\frac{\alpha_{2}\left(\alpha_{4}-\alpha_{1}\right)-\alpha_{4}\left(\alpha_{2}-\alpha_{1}\right) \mathrm{cn}^{2}(r s, M)}{\left(\alpha_{4}-\alpha_{1}\right)-\left(\alpha_{2}-\alpha_{1}\right) \mathrm{cn}^{2}(r s, M)}
$$

where

$$
r=\frac{\sqrt{\left(\alpha_{4}-\alpha_{2}\right)\left(\alpha_{3}-\alpha_{1}\right)}}{4}, \quad M=\sqrt{\frac{\left(\alpha_{4}-\alpha_{3}\right)\left(\alpha_{2}-\alpha_{1}\right)}{\left(\alpha_{4}-\alpha_{2}\right)\left(\alpha_{3}-\alpha_{1}\right)}}
$$

and $\mathrm{cn}(r s, M)$ is the Jacobi Elliptic cosine. The second solution $\widetilde{\kappa}_{d}^{\lambda}(s)$ is obtained by interchanging $1 \leftrightarrow 3$ and $2 \leftrightarrow 4$ in (36). If $Q_{d}(x)$ has two simple roots, then for any $d$ we obtain one solution of (36) which is given by

$$
\begin{aligned}
\kappa_{d}^{\lambda}(s)= & \frac{(p+q)\left(q \alpha_{2}+p \alpha_{1}\right)-2 p q\left(\alpha_{2}-\alpha_{1}\right) \operatorname{cn}(r s, M)}{(p+q)^{2}-(p-q)^{2} \mathrm{cn}^{2}(r s, M)} \\
& +\frac{(p-q)\left(q \alpha_{2}-p \alpha_{1}\right) \mathrm{cn}^{2}(r s, M)}{(p+q)^{2}-(p-q)^{2} \mathrm{cn}^{2}(r s, M)}
\end{aligned}
$$

with

$$
\begin{array}{ll}
p^{2}=\left(\alpha_{2}+\alpha_{1}\right)^{2}+2 \alpha_{2}^{2}-2 \lambda^{2}+4, & q^{2}=\left(\alpha_{2}+\alpha_{1}\right)^{2}+2 \alpha_{1}^{2}-2 \lambda^{2}+4 \\
M=\frac{1}{2} \sqrt{\frac{\left(\alpha_{2}-\alpha_{1}\right)^{2}-(p-q)^{2}}{p q}}, & r=\frac{\sqrt{p q}}{2} .
\end{array}
$$

Let $\kappa(s)$ be a solution to the equation (36) with period $\rho$ and take $\gamma(s)$ the associated curve in $\mathbb{S}^{2}(1)$. According to (28), we know that there exist geographic coordinates in $\mathbb{S}^{2}(1)$,

$$
x(\theta, \phi)=(\cos \theta \sin \phi, \sin \theta \sin \phi, \cos \phi)
$$

such that $\gamma(s)=x(\theta(s), \phi(s))$ and the coordinates of $\gamma(s)$ are determined by

$$
\theta_{s}(s)=\frac{\kappa^{2}-\lambda^{2}}{b\left(d-4(\kappa+\lambda)^{2}\right)}, \quad b^{2}\left(d-4(\kappa+\lambda)^{2}\right)=\sin ^{2} \phi .
$$

It can be shown that $b, d \in \mathbb{R}$ are real constants satisfying $b^{2} d=1$. Hence, following Proposition 15, the closedness condition is now 
Proposition 18. Let $\gamma(s)$ be a curve in $\mathbb{S}^{2}(1)$ corresponding to a periodic solution $\kappa(s)$ of equation (36) with period $\rho$. Then $\gamma(s)$ is a closed $\lambda$-elastic curve, if and only if, its progression angle in one period of its curvature

$$
\Lambda^{\lambda}(d)=\sqrt{d} \int_{0}^{\rho} \frac{\left(\kappa^{2}-\lambda^{2}\right)}{d-4(\kappa+\lambda)^{2}} \mathrm{~d} s
$$

is a rational multiple of $\pi$.

Let the number $\Lambda_{1}$ be defined as

$$
\Lambda_{1}=-4 \lambda \frac{K(M)}{r}+8 \lambda^{2} \int_{\varsigma}^{\lambda} \frac{\mathrm{d} \kappa}{(\kappa+3 \lambda) \sqrt{(\lambda-\kappa)(\kappa-\varsigma)\left((\kappa-u)^{2}+v^{2}\right)}}
$$

where $M$ and $r$ were given previously, $K(M)$ denotes the complete elliptic integral of the first kind, and $\varsigma$ is the only negative root of $\beta^{3}+\lambda \beta^{2}+\beta\left(\lambda^{2}-4\right)-$ $\lambda\left(\lambda^{2}-12\right)=0$. Then, one can obtain the following result [12]

Proposition 19. Assume that $0 \leq \lambda<2 \sqrt{2}$, then for every pair of integer numbers $m, n \in \mathbb{Z}$ satisfying $\left|\frac{\Lambda_{1}}{2 \pi}-\frac{m}{n}\right|<\frac{1}{2}$, there exists a closed $\lambda$-elastic curve $\gamma_{m n}(s)$ in $\mathbb{S}^{2}(1)$. On the other hand, if $\lambda \geq 2 \sqrt{2}$, then for every pair of integer numbers $m, n \in \mathbb{Z}$ satisfying $\frac{m}{n}<0$, there exists a closed $\lambda$-elastic curve $\gamma_{m n}(s)$ in $\mathbb{S}^{2}(1)$. In any of the above cases, $\gamma_{m n}(s)$ closes up after $n$ periods of its curvature and $m$ trips around the equator.

For any $\lambda \geq 2 \sqrt{2}$ there exists a closed "figure eight" shaped $\lambda$-elastic curve in $\mathbb{S}^{2}(1)$. This curve plays an interesting role when analyzing the stable critical points. In order to find the minima of the energy one may consider the second variation formula of $\mathcal{F}^{\lambda}(\gamma)$. It was computed in [10], but it is very difficult to manage unless the critical point has a simple curvature.

For instance, considering critical points of constant curvature, we observe that there are three circles which are critical points: $C_{\eta_{o}}$ with curvature $\kappa=-\lambda$ (which must be obviously a global minimum); $C_{\eta_{1}}$ with curvature $\eta_{1}=\frac{\lambda+\sqrt{\lambda^{2}-8}}{2}$ and $C_{\eta_{2}}$ with curvature $\eta_{2}=\frac{\lambda-\sqrt{\lambda^{2}-8}}{2}$. We have showed that $C_{\eta_{2}}$ is always unstable and that the once covered $C_{\eta_{1}}$ is stable (multiple $m$-covers of this circle $C_{\eta_{1}}^{m}$ are stable provided that $m$ is not too large) [9]. As for the non-constant curvature critical points, we have seen in [12] that a numerical analysis strongly 
suggests that the "eight figure" is stable but we don't have a formal confirmation of this point.

Following a similar procedure, we have investigated the closed critical points for $\mathcal{F}^{\lambda}(\gamma)=\int_{\gamma}(\kappa-\lambda)^{2} \mathrm{~d} s$ in the hyperbolic plane $\mathbb{H}^{2}(-1)$ [13]. The situation here is much richer and new cases appear. Corresponding to the Euler-Lagrange equation, we can encounter three types of the critical curves: rotational, translational and horocyclical type. Nevertheless, we are able to integrate explicitly the EulerLagrange equations in terms of the Jacobi Elliptic functions and, for each case, we can choose suitable adapted coordinates systems where the corresponding closedness conditions is established in terms of the progression angle. We prove that, apart from the horocyclical type, where no closed critical curves exist, there are examples of closed critical curves of the other types. Actually, there are many different examples of rotational type, but we have found only one closed example of translational type. More concretely, denoting by $\delta_{2}$ a real value defined in terms of the roots of the partner polynomial $Q_{d}$ which appear in the hyperbolic case (for details, see [13]), we have

Proposition 20. (1) For any $\lambda>0, d \in\left(-\delta_{2}, 0\right)$, the progression angle $\Lambda^{\lambda}(d)$ moves continuously in $\left(-\delta_{2},-16 \lambda^{2}\right) \cup\left(-16 \lambda^{2}, 0\right)$ and, therefore, there exist infinite many closed critical curves of (35) with rotational symmetry in $\mathbb{H}^{2}(-1)$. (2) For any $\lambda>1, d>0$ ), the progression angle $\Lambda^{\lambda}(d)$ reaches the zero value exactly once, and, therefore, there is a closed "eight figure" critical curve of (35) in $\mathbb{H}^{2}(-1)$ of translational type. (3) For any $\lambda>0$, there exist periodic critical curves of (35) type in $\mathbb{H}^{2}(-1)$ with horocyclical type, but they never close up.

In order to get a better insight of the space of closed critical points, we have checked out the closedness conditions numerically and perform a numerical stability analysis. For example, as we said previously, the "eight figure" shape curve is the only translational type closed critical curve that we have found and numerics suggests that it is, in fact, the only one of this type and, moreover, that it is a candidate to be a local minimum of the energy.

\section{Some Applications}

This section is devoted to show how the generalized elastic curves functionals studied so far have interesting applications in Physics and Mathematics. In Physics, they are useful, for example, to produce fundamental models which play a basic 
role in the understanding of the real world. In Mathematics, apart from their intrinsic interest, they can be used to construct algorithms which are applicable in the context of submanifolds minimizing higher order energies.

\subsection{Models of Relativistic Particles}

The family of energy functionals introduced in Section 3 can be used to provide different models for relativistic particles in Physics. Lagrangians describing relativistic particles, have a long history in Physics. The standard approach considers Lagrangians which depend on higher derivatives of the curve $\gamma$ that represents the worldline of the particle in the spacetime. Investigation of these models in the classical variational setting, gives rise to very complicated nonlinear differential equations which are difficult to analyze. Recent geometric models are intrinsic and describe the particles inside the original space-time where the system is evolving. Thus, if $\gamma$ is a regular curve with $n-1$ Frenet curvature functions, $\kappa_{1}, \kappa_{2}, \cdots, \kappa_{n-1}$, which describes the trajectory of a particle in a "space-time" $\mathbb{M}^{n}$ (we usually will consider $\mathbb{M}^{n}$ as a Riemannian or Lorentzian manifold) the motion of the particle is governed by an action defined by Lagrangian densities depending on the curvatures

$$
\mathcal{F}: \mathcal{H} \rightarrow \mathbb{R}, \quad \mathcal{F}(\alpha)=\int_{\alpha} P\left(\kappa_{1}(s), \kappa_{2}(s), \cdots, \kappa_{n-1}(s)\right) \mathrm{d} s
$$

$\mathcal{H}$ being a certain space of curves. For this kind of Lagrangians, the EulerLagrange equations can be always formulated in terms of the Frenet curvatures $\kappa_{i}$. This is particularly useful if the ambient space is a space-time of constant curvature $G$, because then the Frenet frame provides a complete kinematical description of the particle motion because they are invariant under the group of motions and, once we know the Frenet curvatures $\kappa_{i}$, the trajectory of the particle is totally determined up to rigid motions.

As particular cases one has, for example, that the choice $P\left(\kappa_{1}, \kappa_{2}, \cdots, \kappa_{n-1}\right)=$ $m, m$ being a constant, corresponds to the model for geodesics and describes free fall particles in $\mathbb{M}^{n}$. On the other hand, massless bosons trajectories can be considered as the critical points of the free total curvature $P\left(\kappa_{1}, \kappa_{2}, \cdots, \kappa_{n-1}\right)=$ $m \kappa_{1}$, while the constrained total curvature energy $P\left(\kappa_{1}, \kappa_{2}, \cdots, \kappa_{n-1}\right)=m \kappa_{1}+$ $n$ with $m, n \in \mathbb{R}$, serves to model massive bosons. Tachyonless models of relativistic particles are defined by $\mathcal{F}_{m n p}(\alpha)=\int_{\alpha}\left(m+n \kappa_{1}+p \kappa_{2}\right) \mathrm{d} s$ where $m, n, p \in \mathbb{R}$ (for more details see, for example, [1], [35], [46], [47] [48], [53], [54], [55]). 
In Riemannian and Lorentzian spaces the order one rigidity model

$$
\mathcal{F}_{m}: \mathcal{H} \rightarrow \mathbb{R}, \quad \mathcal{F}_{m}(\gamma)=\int_{\gamma}(\kappa(s)+m) \mathrm{d} s
$$

has been study by different authors [16]. In surfaces, the trajectories of particles are the solutions of the following equations: $m \kappa=\varepsilon_{2} G$, where $G$ is the Gaussian curvature. Hence, trajectories of the free model, i.e., massless model $m=0$, correspond with those curves made up of parabolic points.

In higher dimensions, the free total curvature model is consistent only in three spheres or in anti-de-Sitter three spaces. The dynamics in the three sphere has been previously described (see Table 3 ). On the other hand, in order to completely describe the dynamics in the anti de Sitter three space $\mathbb{A d S}_{3}$, one has to determine the family of helices satisfying:

$$
\left\{(\kappa, \tau) \in \mathbb{R}^{2} ; \tau^{2}-\varepsilon_{2} m \kappa=1\right\}
$$

where $\varepsilon_{2}$ depends on the casual character of the curve. Hence, massive spinning particles in $\mathbb{A d S}_{3}$ defined by the Lagrangian $\mathcal{F}_{m}$, with $m \neq 0$, evolve generating worldlines that are helices in $\mathbb{A d S}_{3}$. The complete solution of the motion equations consists of a one-parameter family of non-congruent helices. Moreover, The moduli space of solutions may be described by three different (but equivalent) pairs of dependent real moduli [16].

The previous analysis can be naturally extended to study models describing relativistic particles where Lagrangian densities depend linearly on both the curvature and the torsion of the trajectories in three-dimensional Lorentzian spacetimes with constant curvature [16], [36]

$$
\mathcal{F}_{\text {mnp }}(\gamma)=\int_{\gamma}(m+n \kappa+p \tau) \mathrm{d} s .
$$

In such a case, the moduli spaces of trajectories are completely and explicitly determined and trajectories are families of Lancret curves which include ordinary helices. Moreover, the geometric integration of the solutions is obtained using B-scrolls, Hopf tubes and the Lancret's program. As a consequence, the moduli subspaces of closed solitons in anti-de Sitter settings can be also obtained.

\subsection{From Membranes in Biophysics to Worldsheets in String Theory}

Investigation of surfaces which are extremal for a free energy which is quadratic in the principal curvatures is relevant in the study of many physical and biophysical problems. They are useful, for example in the theoretical description of 
amphiphilic systems. Well known classes of amphiphiles are tensides or surfactants (used for washing and cleaning purposes) and lipids (the basic components of biomembranes). The physics of amphiphilic systems is mostly determined by their interfaces. In binary systems, amphiphiles usually self-assemble into bilayer structures which are called fluid membranes. Thus, embedded surfaces $S$ in Euclidean space $\mathbb{R}^{3}$ are considered not so much as a geometric object but as an idealized model for the interfaces or middle surfaces occurring in real materials: open or closed lipid bilayers (membranes and vesicles); surfactant films; thin elastic plates, etc...

The free energy of an amphiphilic system can be written as a functional of its interfacial geometry and the shape of the interface $S$ is determined by the mechanical equilibrium of the free energy. The interface's elastic properties suggest that the free energy of $S$ is controlled not only by the tension, but also by the curvature. Moreover, in a "linear" elasticity theory [37], Hooke's law indicates that the free energy of a surface, $\mathcal{E}(S)$, is quadratic in the principal curvatures. Then, we may assume that the Lagrangian controlling the energy is given by $\widetilde{\Phi}\left(\kappa_{1}, \kappa_{2}\right)=\Phi(H, K)=a+b\left(H-c_{o}\right)^{2}-c K$, where $K$ is the Gaussian curvature of the interface $S, H$ is the Mean curvature and $a, b, c \in \mathbb{R}$ are physical constants.

Thus, we obtain a simple geometric model (a particular simple case was first studied by S. Germain around 1810 in her analysis of the elastic plates) according to which the elastic energy $\mathcal{E}(S)$ of a surface $S$ embedded in a three-space form can be defined by

$$
\mathcal{E}(S)=\int_{S}\left(a+b\left(H-c_{o}\right)^{2}+c K\right) \mathrm{d} A
$$

where $a, b, c, c_{o} \in \mathbb{R}$ and $\mathrm{d} A$ is the area element of the surface $S$. These constants represent material quantities as surface tension, elastic moduli,...etc, and $c_{o}$ is the spontaneous curvature related to a initial state asymmetry in the two faces of the bilayer. Classically, this type of models have been considered by S. Poisson 1812, G. Kirchhoff 1850, A. Love 1906 and, more recently, by P. Canhman [19], W. Helfrich [26] within a biophysical context. T. Thomsem, H. Hopf and T. Willmore studied them from a mathematical point of view [61].

Actually, analogous energies can be considered in any real space form and, for suitable choices of the parameters involved in (38), the family of critical points includes distinguished members as surfaces of constant mean curvature (soap bubbles), minimal surfaces (soap films),...etc. For instance, if $b>0$, we can suppose that an evenness assumption is taken, that is $c_{o}=0$, and, by scaling, that $b=1$. 
We also assume that $a$ is equal to the curvature of the ambient real three-space form, so $a=-1,0,1$. Then, the energy in this case, commonly denoted by $\mathcal{W}(S)$, is known as the Willmore functional and their critical points are called Willmore surfaces (membranes).

As we have said, an equilibrium elastic surface $S$ constitutes a minimizer or, more generally, a critical point of $\mathcal{E}(S)$ subject to the constraints of the problem. For example, if $S$ is a closed surface of $\mathbb{R}^{3}$, one may consider the shape energy functional $\mathcal{M}(S)$ defined by

$$
\mathcal{M}(S)=\mathcal{E}(S)+d \int_{\Omega} \mathrm{d} V
$$

where $d \in \mathbb{R}$ and $\mathrm{d} V$ is the volume element of the region $\Omega$ enclosed by $S$. Moreover, the last term of $\mathcal{M}(S)$ either takes account of a constraint on constant volume or it can represent actual work. When the ambient space is $\mathbb{R}^{3}$, the functional is of fundamental importance in understanding the role of bending elasticity for both equilibrium shapes and for shape fluctuations of membranes. Critical points of $\mathcal{M}(S)$ serve as models for biological membranes ([19], [26], [37], [42] [51]) which are called vesicles if they are closed. Closely related models are very useful to study other important physical entities as liquid crystals, polymers and filaments [24], [31], [34], [56] and [62]. The static equilibrium shape of a interface $S$ is determined by the condition $\delta \mathcal{E}=0$ and the variational problem leads not only to the Euler-Lagrange equations

$$
b\left\{\triangle H+2 H\left(H^{2}-K\right)\right\}-2\left(a+b c_{o}^{2}\right) H+2 b c_{o} K=0
$$

where $\triangle$ is the Laplacian of $S$, but also to certain specific intrinsic, or natural, boundary conditions [49]

$$
\begin{array}{r}
-b \frac{\partial H}{\partial n}-c\left\{\frac{\partial \tau}{\partial s}+\frac{\partial^{2} \vartheta}{\partial s^{2}}\right\} \\
b\left(H-c_{o}\right)-c \kappa_{n} \\
-a+b\left(H-c_{o}\right)^{2} c_{o} K
\end{array}
$$

where $\kappa_{n}, n$ are the normal curvature and the interior normal of $\partial S$ in $S, \tau$ is the torsion of $\partial S$ in $\mathbb{R}^{3}$, and $\vartheta=\angle(\mathbb{N}, n), \mathbb{N}$ being the unit normal to $S$.

Often the interface separates two media of prescribed volumes, so we must add to the energy a volume constraint as in (39). The Euler-Lagrange equation is now

$$
b\left\{\triangle H+2 H\left(H^{2}-K\right)\right\}-2\left(a+b c_{o}^{2}\right) H+2 b c_{o} K-d=0 .
$$


Obviously, the boundary conditions will have to be complemented as well. The equation (42) is a nonlinear partial differential equation of fourth order for $x$, the position vector of $S$, but using the Beltrami's equation

$$
\triangle x=2 H \mathbb{N}
$$

we see that (42) can be written in the form of four differential equations of second order (three, namely (43), for $x$, and one, namely (42), for the mean curvature $H$ ). Hence, the shape problem for membranes lead us to a boundary value problem for variational integrals: determination of minimizing or stationary surfaces for the energy functional in the class of all surfaces of a prescribed topological type (subject or not to a volume constraint) and with boundaries on fixed curves (Plateau type) or on prescribed surfaces (free boundary).

For mathematicians the most central question is the existence proof of stationary surfaces. The existence and uniqueness of minimizers of $\mathcal{E}(S)$ of a certain topological class is still unknown, although there are some partial results in particular cases. It is also not known whether the minimizer is symmetric in any sense. On the mathematical level the attending problems are really complicated [49].

On the other hand, Physicists and Biophysicists are more interested in explicit solutions to (42), since they can be used to derive physical properties of the corresponding system. However, very few explicit solutions are known. As far as closed surfaces are concerned (vesicles), we have of course the spheres, certain anchor rings and some cyclides of Dupin [62]. But even, for surfaces of revolution, where the problem simplifies notably to a one-variable equation, the whole set of solutions are not know (however, some particular non-trivial solutions have been found [45], [62]). There have been extensive numerical investigations of the solution surfaces of (42), but generally restricted to surfaces with rotational symmetry [57], [58]. Vesicles of more complex topology have been also analyzed [29], [58] and the numerical solutions have been successfully tested in the laboratory [43], [44].

Fortunately, elastic curves can be used to construct solutions to the Euler-Lagrange equation (42), at least for suitable choices of the parameters. Observe that the one-dimensional version of membranes are the elastic curves. J. Nitsche proved in [49] that, under certain boundary conditions, cylindrical membranes in $\mathbb{R}^{3}$ are cylinders shaped on plane elastic curves, which shows a first relationship between membranes and elasticae. On the other hand, the simplest type of elastic energy is the bending energy or Willmore energy. Critical points of the bending energy

$$
\mathcal{W}(S)=\int_{S} H^{2} \mathrm{~d} A
$$


are called Willmore surfaces. A very important property of the Willmore energy is its conformal invariance. This fact has been used by J. Langer and D. Singer [40] to show that the surfaces of revolution in $\mathbb{R}^{3}$ which are Willmore membranes are precisely those shaped on the elastic curves of $\mathbb{H}^{2}(-1)$. Also, Hertrich-Jeromin has shown [27] that a Willmore channel surface is Moebius equivalent either to a cone shaped on a free elastic curve of $\mathbb{S}^{2}(1)$, or to a cylinder shaped on a free elastic curve of $\mathbb{R}^{2}$, or to a surface of revolution shaped on a free elastic curve of $\mathbb{H}^{2}(-1)$. Therefore, explicit examples of elastic curves would provide explicit solutions to (42).

In [60] J. Weiner showed that minimal surfaces of real space forms are examples of Willmore surfaces. Consequently, he used the conformal invariance, the stereographic projection and the Lawson minimal examples in $\mathbb{S}^{3}$, to produce Willmore surfaces of any genus in $\mathbb{R}^{3}$. The first examples of Willmore membranes in $\mathbb{R}^{3}$ which did not come from minimal surfaces of $\mathbb{S}^{3}(1)$ were constructed using Hopf Tori shaped on the elastic curves of $\mathbb{S}^{2}(1 / 2)$ by U. Pinkal [52]. In a similar way closed vesicles in $\mathbb{S}^{3}$ may be produced by lifting closed elasticae in $\mathbb{S}^{2}$ which are circular at rest [9].

We have seen so far, how elastic curves are useful to construct explicit examples of Willmore surfaces. But Willmore surfaces in turn (pure elastic surfaces) have also applications to String Theory. In a string theory, distinguished curves dynamically evolve in a gravitational space and sweep out surfaces, worldsheets, that are solutions to the field equations associated with a string action. A natural extension of this idea leads to the notion of branes and worldvolumes. The first and simplest choice of an action, in order to construct a bosonic string theory, was the Nambu-Goto action which is defined in terms of the area of the worldsheets. Thus, minimal surfaces in the corresponding gravitational space are the natural worldsheets in the Nambu-Goto bosonic string theory. A more interesting choice is that known as the Kleinert-Polyakov action, where the extrinsic geometry of worldsheets is introduced in the string action. Since the most important extrinsic invariant is the mean curvature, this idea leads naturally to the following choice for the string action

$$
\mathcal{P K}(S)=\mu \int_{S} \mathrm{~d} A+\nu \int_{S} H^{2} \mathrm{~d} A
$$

which is another version of the Willmore energy (44). For more details, see, for example, [15], [17], [33]. 


\subsection{Chen-Willmore Submanifolds}

Generalized elasticae have also applications to the theory of Chen-Willmore submanifolds [20], [21]. In the early seventies, B-Y. Chen extended the ThomsemWillmore functional to any submanifold $\mathbb{M}^{n}$ of any Riemannian manifold $\mathbb{N}^{m}$. He defined Chen-Willmore functional

$$
\mathcal{C W}(M)=\int_{M}\left(H^{2}-\tau_{e}\right)^{\frac{n}{2}} \mathrm{~d} v
$$

$H$ and $\tau_{e}$ being the mean curvature and the extrinsic scalar curvature of $\mathbb{M}^{n}$, respectively. The energy (45) is also conformally invariant and its critical points are known as Chen-Willmore submanifolds. If $n=2$ and $\mathbb{N}^{m}=\mathbb{R}^{3}$ it coincides with the classical Willmore functional.

An important fact in this theory is to develop methods for the construction of examples of Chen-Willmore submanifolds. Note that, in contrast with the surfaces situation, a minimal submanifold of the sphere is not necessarily a Chen-Willmore submanifold [25], which makes it considerably harder to search for explicit examples. Following Pinkal's idea [52], by combining the conformal invariance of the Chen-Willmore energy with the Palais' Symmetric Criticality Principle [50], we gave a quite general procedure to construct Chen-Willmore submanifolds in warped product Riemannian manifolds [3], [8].

A useful version of Palais' Principle for our purposes can be formulated as follows. Take a manifold $\mathbb{N}$ and a group $G$ which acts by diffeomorphisms on it. Consider a $G$-invariant functional $\mathcal{W}: \mathbb{N} \rightarrow \mathbb{R}$, i.e., $\mathcal{W}(a \cdot \varphi)=\mathcal{W}(\varphi)$, for all $a \in$ $G$ and define the following sets: 1) $\mathbb{N}_{G}=\{\varphi \in \mathbb{N} ; a \cdot \varphi=\varphi$, for all $a \in G\}$ is the set symmetric points; 2) $\Sigma$ represents the critical points of $\mathcal{W}: \mathbb{N} \rightarrow \mathbb{R}$ and 3) $\Sigma_{G}$ denotes the critical points of the restriction of $\mathcal{W}$ to the set $\mathbb{N}_{G}$ of symmetric points. Then, if $G$ is compact, we have that $\mathbb{N}_{G}$ is a submanifold of $\mathbb{N}$ and Palais' principle says that $\Sigma \cap \mathbb{N}_{G}=\Sigma_{G}$.

In [3] we have obtained the following result

Theorem 21. Let $(M, g)=M_{1} \times_{f} M_{2}$ be a warped product where $\left(M_{2}, g_{2}\right)$ is a compact homogeneous space of dimension $n_{2}$. Let $\gamma$ be a closed curve immersed in $\left(M_{1}, g_{1}\right)$. The submanifold $N=\gamma \times{ }_{f} M_{2}$ is a Chen-Willmore submanifold in $(M, g)$ if and only if $\gamma$ is a $n_{2}$-generalized elastica in $\left(M_{1}, \frac{1}{f^{2}} g_{1}\right)$.

The main point here is that we can relate the Chen-Willmore variational problem to that of hyperelastic curves in the conformal structure on the base space. It 
explains also the Willmore cylinders shaped on plane elastica, the Willmore Hopf Tori shaped on spherical elastica and the Willmore surfaces of revolution shaped on hyperbolic elastica that we have mentioned before.

In [8] we used a different application of Palais' principle to characterize ChenWillmore rotational hypersurfaces of $\mathbb{R}^{n+1}$ and $\mathbb{S}^{n+1}$, in terms of the closed free $\mathrm{n}$-elastic curves of the hyperbolic plane $\mathbb{H}^{2}(-1)$. Hence, by using the classification results and methods described in the previous section, we produced the first examples of Chen-Willmore hypersurfaces of $\mathbb{R}^{n+1}$ and $\mathbb{S}^{n+1}(1)$, which are not in the conformal class of the standard examples. In order to do that, we proved that there exist periodic solutions to the Euler-Lagrange equation for hyperelastic curves $\mathbb{H}^{2}(-1)$ and give a qualitative description of the non-constant curvature closed hyperelastic ( $n$-elastic) curves: they are convex curves travelling along a circle $\epsilon_{n}$, which oscillate between two concentric circles and close up after an integer number of trips around $\epsilon_{n}$. Getting concrete examples of rotational ChenWillmore hypersurfaces would require, first to solve explicitly the Euler-Lagrange equations, and then to quantify the closedness condition. Although this task does not seem to be possible in general, it has been done for $n=2$ by J. Langer and $\mathrm{D}$. Singer [38] and for $n=3$ by J. Arroyo, M. Barros and O. Garay [6]. In fact, we proved that the Euler-Lagrange equation of three-elastic curves in $\mathbb{H}^{2}(-1)$ can be explicitly integrated and the corresponding Frenet equations can be integrated by quadratures. In this way, we found a rationally dependent family of curves which fulfilled the closedness condition and, therefore, they provide the required explicit examples of Chen-Willmore hypersurfaces in $\mathbb{R}^{4}$.

\section{Acknowledgements}

Supported by grants GIC07/58-IT-256-07 of Gobierno Vasco and MTM200761990 of Ministerio de Educación y Ciencia. Spain

\section{References}

[1] Arreaga G., Capovilla R. and Guven J., Frenet-Serret Dynamics, Class. Quantun Grav. 18 (2001) 5065-5083.

[2] Arroyo J., Presión Calibrada Total, Estudio Variacional y Aplicaciones al Problema de Chen-Willmore, PhD Thesis. Univ. del País Vasco, Leioa, Spain, 2001. 
[3] Arroyo J., Barros M. and Garay O., Willmore-Chen Tubes on Homogeneous Spaces in Warped Product Spaces, Pacific J. Math. 188 (1999) 201-207.

[4] Arroyo J., Barros M. and Garay O., Some Examples of Critical Points for the Total Mean Curvature Functional, Proc. Edinburgh Math. Soc. 43 (2000) 587-603.

[5] Arroyo J., Barros M. and Garay O., Holography and Total Charge, Journal of Geometry and Physics 742 (2001) 1-8.

[6] Arroyo J., Barros M. and Garay O., Relativistic Particles with Rigidity Generating Non-Standard Examples of Willmore-Chen Hypersurfaces, J. Phys. A: Math. Gen. 35 (2002) 6815-6824.

[7] Arroyo J., Barros M. and Garay O., Models of Relativistic Particles with Curvature and Torsion Revisited, Gen. Rel. Gravit. 362004 1441-1451.

[8] Arroyo J., Barros M. and Garay O., Closed Free Hyperelastic Curves in the Hyperbolic Plane and Chen-Willmore Rotational Hypersurfaces, Israel J. Math. 138 (2003) 171-187.

[9] Arroyo J and Garay O J, Hopf Vesicles in $\mathbb{S}^{3}(1)$, Contemporary Math. 288 (2001) 258-262.

[10] Arroyo J., Garay O. and Mencía J., Closed Generalized Elastic Curves in $\mathbb{S}^{2}(1)$, J. Geom. Phys. 48 (2003) 339-353.

[11] Arroyo J., Garay O. and Mencía J., Extremals of Curvature Energy Actions on Spherical Closed Curves, J. Geom. Phys. 51 (2004) 101-125.

[12] Arroyo J., Garay O. and Mencía J., Elastic Circles in 2-Spheres, J. Phys. A: Math. Gen. 39 (2006) 2307-2324.

[13] Arroyo J., Garay O. and Mencía J., Elastic Curves in the Hyperbolic Plane, Preprint, 2008.

[14] Barros M., General Helices and a Theorem of Lancret, Proc. A.M.S. 125 (1997) 1503-1509.

[15] Barros M., Simple Geometric Models with Applications to Physics, In: Curvature and Variational Modeling in Physics and Biophysics, O. Garay, E. García-Río and R. Vázquez-Lorenzo (Eds), AIP Conference Proceedings 1002, Melville-New York, 2008, pp 71-113.

[16] Barros M., Ferrandez A., Javaloyes M. and Lucas P., Relativistic Particles with Rigidity and Torsion in $D=3$ Spacetimes, Class. Quantun Grav. 35 (2005) 489-513.

[17] Barros M., Ferrández A. and Lucas P., Conformal Tension in String Theories and M-Theory, Nucl. Phys. B 584 (2000) 719-748. 
[18] Bryant R. and Griffiths Ph., Reduction for Constrained Variational Problems and $\int \frac{1}{2} k^{2} d s$, Amer. J. Math. 108 (1986) 525-570.

[19] Canham P., The Minimum Energy of Bending as a Possible Explanation of the Biconcave Shape of the Human Red Blood Cell, J. Theor. Biol. 26 (1970) 61-81.

[20] Chen B.Y., Some Conformal Invariants of Submanifolds and Their Applications, Boll. Un. Mat. Ital. 6 (1974) 380-385.

[21] Chen B.Y., Total Mean Curvature and Submanifolds of Finite Type, World Scientific, Singapore, 1984.

[22] Djondjorov P., Hadzhilazova M., Mladenov I. and Vassilev V., Explicit Parametrization of the Euler's Elastica, In: Geometry, Integrability and Quantization, I. Mladenov (Ed), Softex, Sofia, 2008, pp 175-186.

[23] Euler L., Methodus Inveniendi Lineas Curvas Maximi Minimive Proprietate Gaudentes, Sive Solutio Problematis Isoperimetrici Lattisimo Sensu Accepti, Bousquet, Lausannae et Genevae E 65A., O.O. Ser. I, vol 24, 1744.

[24] Goldstein R. and Langer S., Nonlinnear Dynamics of Stiff Polymers, Phys. Rev. Lett. 75 (1995) 1094-1097.

[25] Guo Z., Li H. and Wang Ch., The Second Variational Formula for Willmore Submanifolds in Sspn, Results Math., 40 (2001) 205-225.

[26] Helfrich W., Elastic Properties of Lipid Bilayers: Theory and Possible Experiments, Z. Naturforsch 28c (1973) 693-703.

[27] Hertrich-Jeromin U., Introduction to Moebius Differential Geometry, London Mathematical Soc. Lecture Note Series 300, Cambridge Univ. Press., Cambridge, 2003.

[28] Hopf H., Über Die Abbildungen der Dreidimensionalen Sphäre auf die Kugelfä̈che, Math. Ann. 104 (1931) 637-665.

[29] Jülicher F., The Morphology of Vesicles of higher Topological Genus: Conformal Degeneracy and Conformal Modes, J. Phys. II France 6 (1996) 17971824.

[30] Jurdjevic V., Non-Euclidean Elastica, Amer. J. Math. 117 (1995) 93-124.

[31] Kamien R., The Geometry of Soft Materials: A Primer, Rev. Mod. Phy. 74 (2002) 953-971.

[32] Kawakubo S., Kirchhoff Elastic Rods in Three-Dimensional Space Forms, J. Math. Soc. Japan 60 (2008) 551-582.

[33] Kholodenko A. and Nesterenko V., Clasical Dynamics of the Rigid String from the Willmore Funcional, J. Geom. Phys. 16 (1995) 15-26. 
[34] Kierfeld J., Baczinsky K., Gutjahr P and Lypowsky R., Semiflexible Polymers and Filaments: From Variational Problems to Fluctuations, In: Curvature and Variational Modeling in Physics and Biophysics, O. Garay, E. García-Río and R. Vázquez-Lorenzo (Eds), AIP Conference Proceedings 1002, Melville-New York, 2008, pp 151-185.

[35] Kuznetsov Y. and Plyushchay M., Tachyonless Models of Relativistic Particles with Curvature and Torsion, Phys. Lett. B 297 (1992) 49-54.

[36] Kuznetsov Y. and Plyushchay M., The Model of the Relativistic Particle with Curvature and Torsion, Nucl. Phys. B 389 (1993) 181-208.

[37] Landau L. and Lifshitz E., Theory of Elasticity, (Course of Theoretical Physics, Vol 7, Butterworth-Heinemann, Boston, MA, 1986.

[38] Langer J. and Singer D., The Total Squared Curvature of Closed Curves, J. Diff. Geom. 20 (1984) 1-22.

[39] Langer J. and Singer D., Knotted Elastic Curves in $\mathbb{R}^{3}$, J. London Math. Soc. 30 (1984) 512-520

[40] Langer J. and Singer D., Curves in the Hyperbolic Plane and Mean Curvature of Tori in 3-Space, Bull. London Math. Soc. Ann. 16 (1984) 531-534.

[41] Langer J. and Singer D., Lagrangian Aspects of the Kirchhoff Elastic Rod, SIAM Rev. 38 (1996) 605-618.

[42] Lipowsky R., The Conformation of Membranes, Nature 349 (1991) 475-481.

[43] Michalet X. and Bensimon D., Vesicles of Toroidal Topology: Observed Morphology and Shape Transformations, J. Phys. II France 5 (1995) 263287.

[44] Michalet X. and Bensimon D., Observation of Stable Shapes and Conformal Diffusion in Genus 2 Vesicles, Science 269 (1995) 666-668.

[45] Mladenov I., New Solutions of the Shape Equation, Eur. Phys. J. B 29 (2002) 327-330.

[46] Nersessian A., Massive 4d Particle with Torsion and Conformal Mechanics, Phys. Lett. B 473 (2000) 94-101.

[47] Nesterenko V., On The Model of the Relativistic Particle with Curvature and Torsion, J. Math. Phys. 34 (1993) 5589-5595.

[48] Nesterenko V., Feoli A. and Scarpetta G., Dynamics of Relativistic Particles with Lagrangians Dependent on Acceleration, J. Math. Phys. 36 (1995) 5552-5564.

[49] Nitsche J., Boundary Value Problems for Variational Integrals Involving Surface Curvatures, Q. Appl. Math. 60 (1993) 363-387. 
[50] Palais R., Critical Point Theory and the Minimax Principle, Global Analysis, Proc. Sympos. Pure Math. 15, 1970, pp. 185-212

[51] Palmer B., Variational Problems which are Quadratic in the Surface Curvatures, In: Curvature and Variational Modeling in Physics and Biophysics, O. Garay, E. García-Río and R. Vázquez-Lorenzo (Eds), AIP Conference Proceedings 1002, Melville-New York, 2008, pp 71-113.

[52] Pinkall U., Hopf Tori in $\mathbb{S}^{3}$, Invent. Math. 81 (1995) 379-386.

[53] Plyushchay M., Massless Point Particle with Rigidity, Mod. Phys. Lett. A 4 (1989) 837-847.

[54] Plyushchay M., Massless Particle with Rigidity as a Model for the Description of Bosons and Fermions, Phys. Lett. B 243 (1990) 383-388.

[55] Plyushchay M., The Model of the Relativistic Particle with Torsion, Nuclear Phys. B 362 (1991) 54-72.

[56] Santangelo Ch., The Geometry and Topology of Liquid Crystals, In: Curvature and Variational Modeling in Physics and Biophysics, O. Garay, E. García-Río and R. Vázquez-Lorenzo (Eds), AIP Conference Proceedings 1002, Melville-New York, 2008, pp 114-150.

[57] Seifert U., Berndl K. and Lipowsky R., Shape Transformations of Vesicles: Phase Diagram for Spontaneous Curvature and Bilayer Coupling Model, Phys. Rev. A. 44 (1991) 1182-1202.

[58] Seifert U., Configurations of Fluid Membranes and Vesicles, Adv. Phys. 46 (1997) 13-137.

[59] Singer D., Lectures on Elastic Curves and Rods, In: Curvature and Variational Modeling in Physics and Biophysics, O. Garay, E. García-Río and R. Vázquez-Lorenzo (Eds), AIP Conference Proceedings 1002, Melville-New York, 2008, pp 3-32.

[60] Weiner J., On a Problem of Chen, Willmore, et al., Indiana Univ. Math. J. 27 (1978) 19-35.

[61] Willmore T., Total Curvature in Riemannian Geometry, E Horwood Limited, Chicester, 1982.

[62] Zhong-Can O-Y., Ji-Xing L. and Yu-Zhang X., Geometric Methods in the Elastic Theory of Membranes in Liquid Crystal Phases, World Scientific, Singapore, 1999. 
Óscar J. Garay

Departamento de Matemáticas

Facultad de Ciencia y Tecnología

Universidad del País Vasco

Aptdo 644, 48080, Bilbao

SPAIN

E-mail address: oscarj.garay@ehu.es 